Published in final edited form as:

J Alzheimers Dis. 2020 ; 77(4): 1579-1594. doi:10.3233/JAD-200187.

\title{
Brainstem volumetric integrity in preclinical and prodromal Alzheimer's disease
}

\author{
Shubir Dutt ${ }^{\mathrm{a}, \mathrm{b}}$, Yanrong $\mathrm{Li}^{\mathrm{c}}$, Mara Mather, ${ }^{\mathrm{a}, \mathrm{b}}$, Daniel A. Nation ${ }^{\mathrm{c}, \mathrm{d},{ }^{*}}$ on behalf of Alzheimer's \\ Disease Neuroimaging Initiative \\ aDepartment of Psychology, University of Southern California, Los Angeles, CA, USA \\ bDavis School of Gerontology, University of Southern California, Los Angeles, CA, USA \\ Institute for Memory Impairments and Neurological Disorders, University of California, Irvine, \\ Irvine, CA, USA \\ ${ }^{\mathrm{d} D e p a r t m e n t}$ of Psychological Science, University of California, Irvine, Irvine, CA, USA
}

\begin{abstract}
Background: Neuropathological studies have suggested the tau pathology observed in Alzheimer's disease (AD) originates in brainstem nuclei, but no studies to date have quantified brainstem volumes in clinical populations with biomarker-confirmed mild cognitive impairment (MCI) or dementia due to $\mathrm{AD}$ or determined the value of brainstem volumetrics in predicting dementia.
\end{abstract}

Objective: The present study examined whether MRI-based brainstem volumes differ among cognitively normal older adults and those with $\mathrm{MCI}$ or dementia due to $\mathrm{AD}$ and whether preclinical brainstem volumes predict future progression to dementia.

Methods: Alzheimer's Disease Neuroimaging Initiative participants $(\mathrm{N}=1,629)$ underwent baseline MRI scanning with variable clinical follow-up (6-120 months). Region of interest and voxel-based morphometric methods assessed brainstem volume differences among cognitively normal $(\mathrm{n}=814)$, MCI $(\mathrm{n}=542)$, and $\mathrm{AD}(\mathrm{n}=273)$ participants, as well as subsets of CSF biomarker-confirmed MCI $(\mathrm{n}=203)$ and $\mathrm{AD}(\mathrm{n}=160)$ participants.

Results: MCI and AD cases showed smaller midbrain volumes relative to cognitively normal participants when normalizing to whole brainstem volume, and showed smaller midbrain, locus coeruleus, pons, and whole brainstem volumes when normalizing to total intracranial volume. Cognitively normal individuals who later progressed to AD dementia diagnosis exhibited smaller

\footnotetext{
Corresponding author: Daniel A. Nation, Ph.D., Associate Professor, Department of Psychological Science, University of California Irvine, 4544 Social and Behavioral Sciences Gateway, Irvine, CA 92697-7085, 949-824-9339, dnation@uci.edu.

* Data used in preparation of this article were obtained from the Alzheimer's Disease Neuroimaging Initiative (ADNI) database (adni.loni.usc.edu). As such, the investigators within the ADNI contributed to the design and implementation of ADNI and/or provided data but did not participate in analysis or writing of this report.

DISCLOSURE STATEMENT

The authors have no conflicts of interest to report.

SUPPLEMENTARY MATERIAL

Supplementary material is available in the electronic version of this article.
} 
baseline midbrain volumes than individuals who did not develop dementia, and voxel-wise analyses revealed specific volumetric reduction of the locus coeruleus.

Conclusion: Findings are consistent with neuropathological observations of early AD-related pathology in brainstem nuclei and further suggest the clinical relevance of brainstem substructural volumes in preclinical and prodromal AD.

\section{Keywords}

Alzheimer's disease; Biomarkers; Brainstem; Cognitive aging; Locus coeruleus; Mild cognitive impairment; Neuroimaging; Magnetic resonance imaging

\section{INTRODUCTION}

Neuropathological studies have suggested tau protein-related Alzheimer's disease (AD) pathophysiological processes begin in midbrain and pontine nuclei and precede any observable cortical changes [1,2]. The classic Braak staging of AD pathology was subsequently updated to include precortical stages whereby neurofibrillary tangles first appear in brainstem nuclei and later spread to transentorhinal, hippocampal, and neocortical regions in a stereotypical fashion $[3,4]$. However, there has been recent debate regarding whether brainstem nuclei represent the actual origin sites of tau seeding activity or simply the earliest regions showing phospho-tau signal [5-8]. Thus, identification of an origin site for tau seeding in $\mathrm{AD}$ remains controversial. Despite strong evidence from postmortem autopsy studies, it remains unclear whether corresponding pathological abnormalities may be detected with in vivo brain MRI and whether observable brainstem pathology is clinically relevant for cognitive impairment and dementia.

A growing number of studies have identified progressive accumulation of neurofibrillary tangle pathology in midbrain (e.g., raphe nuclei, substantia nigra) and pontine (e.g., locus coeruleus, pedunculopontine nucleus) nuclei with increasing Braak stage, implicating the disruption of ascending neurotransmitter systems in the manifestation of atypical AD symptoms such as sleep-wake dysregulation, attentional/dysexecutive deficits, and neuropsychiatric abnormalities [9-11]. These histopathological approaches are supported by in vivo neuroimaging studies observing reduced midbrain and pontine volumes in clinicallydiagnosed AD compared to cognitively normal older adults [12-16]. A shape analysis of the brainstem in $\mathrm{AD}$ patients and normal controls demonstrated deformation of a dorsal rostral brainstem region, and a recent voxel-wise study of the brainstem in $\mathrm{AD}$ and controls similarly showed differences in the dorsal rostral brainstem $[12,16]$. However, these studies were limited by relatively small sample sizes and a lack of sub-regional analyses. Furthermore, brainstem volumetric differences remain unexamined in biomarker-confirmed $\mathrm{AD}$ populations, the prodromal mild cognitive impairment (MCI) stage of disease, or the asymptomatic preclinical stage in cognitively normal individuals who eventually develop $\mathrm{AD}$ dementia. The present study aimed to address the dearth of knowledge regarding in vivo brainstem imaging in $\mathrm{AD}$ by quantifying brainstem subregions in a large, longitudinal study of MCI and $\mathrm{AD}$ dementia patients, conducting a sub-study in biomarker-confirmed $\mathrm{AD}$ cases, and examining the potential utility of brainstem volumetrics in predicting development of $\mathrm{AD}$ dementia in initially asymptomatic individuals. 


\section{MATERIALS AND METHODS}

\section{Study Participants}

Participant data were drawn from the Alzheimer's Disease Neuroimaging Initiative (ADNI) database (adni.loni.usc.edu). The ADNI began in 2003 to test whether serial MRI, positron emission tomography, biofluid markers, and clinical and neuropsychological assessment can be combined to measure progression of $\mathrm{MCI}$ and early $\mathrm{AD}$. For up-to-date information, see www.adni-info.org. Inclusion criteria for ADNI consisted of participants ages 55-90 years (inclusive), available study partner capable of accompanying participant to visits, Geriatric Depression Scale score < 6, Hachinski Ischemic Score $\leq 4$, stability of permitted medications for 4 weeks, adequate visual and auditory abilities for neuropsychological testing, adequate general health with no diseases expected to interfere with study participation, minimum of $6^{\text {th }}$ grade education or equivalent work history, and fluency in English or Spanish. Exclusion criteria consisted of significant co-morbid neurological disease, history of substance abuse within the past 2 years, and history of significant head trauma. All participants received baseline clinical diagnoses of cognitively normal $(\mathrm{CN})$, $\mathrm{MCI}$, or AD dementia according to ADNI diagnostic criteria, which have been previously described [17]. This study was conducted in compliance with the Declaration of Helsinki and was approved at all sites by local Institutional Review Boards. All participants or legal representatives of participants gave written informed consent prior to participation in the study. For the present study, participant data consisted of 1,629 older adults enrolled in ADNI-1, ADNI-GO, or ADNI-2 with complete baseline data for all variables of interest (demographics, neuropsychological testing, baseline structural MRI). Age, sex, years of education, and apolipoprotein $(A P O E) \varepsilon 4$ carrier status were included as demographic variables. Further information regarding $A P O E \varepsilon 4$ genotyping is online (http:// adni.loni.usc.edu/data-samples/data-types/genetic-data/).

\section{Cluster Analysis}

Due to the previously noted susceptibility of ADNI MCI diagnoses to false positives, all participants with baseline ADNI diagnoses of MCI were entered into a cluster analysis to resolve potential misclassifications [18-20]. First, a consistently cognitively normal reference group was formed from participants who were ADNI-diagnosed CN and remained $\mathrm{CN}$ for the length of their participation in the study $(n=383)$. Next, linear regression models were run within this group to predict cognitive performance from age and education for six neuropsychological tests (Rey Auditory Verbal Learning Test delayed memory recall, Rey Auditory Verbal Learning Test delayed memory recognition, Animal fluency, Boston Naming Test, Trail Making Test Parts A \& B) across three cognitive domains (memory, language, executive function). Resulting regression coefficients were then used to calculate expected performance of MCI participants on the six neuropsychological tests based on their age and education. Finally, age- and education-adjusted z-scores (calculated based on their observed versus expected performance) were used in a hierarchical cluster analysis (Ward's method \& forced 4-cluster solution) in line with prior studies to reclassify MCI participants into four previously described diagnostic groups: a cluster-derived cognitively normal group, amnestic MCI, dysnomic MCI, and dysexecutive MCI. The cluster-derived cognitively normal group was combined with ADNI-diagnosed cognitively normal individuals to form 
the $\mathrm{CN}$ group for the present study, while the three MCI subtypes were combined into a single neuropsychologically-confirmed MCI group. ADNI-diagnosed AD dementia represented the $\mathrm{AD}$ group.

\section{Neuroimaging Acquisition and Analyses}

ADNI participants underwent MRI scanning on Siemens, GE, or Phillips scanners at 1.5T or 3T magnet strength. T1-weighted structural images were acquired using either a volumetric magnetization prepared rapid gradient-echo sequence (MPRAGE) or a sagittal 3D inversionrecovery prepared spoiled gradient echo imaging pulse sequence (IR-SPGR). Specific parameters for each sequence are available to view online (http://adni.loni.usc.edu/methods/ mri-tool/mri-analysis/). Combining data from 1.5T and 3T magnetic field strengths has been previously shown to be feasible by the ADNI investigators and independent researchers, and we accordingly merged MRI scans from both $1.5 \mathrm{~T}$ and $3 \mathrm{~T}$ field strengths [21,22]. For all study participants, baseline T1-weighted images were first downloaded from the ADNI database (http://adni.loni.usc.edu) in raw NIfTI format prior to any processing. Using the "Display" function in SPM12 (http://www.fil.ion.ucl.ac.uk/spm/) within MATLAB (MATLAB R2018a, MathWorks Inc., Natick, MA, USA) on macOS, each T1-weighted image was individually checked for image quality and manually aligned and rotated to ensure AC-PC (anterior commissure-posterior commissure) alignment. Aligned images were processed through the voxel-based morphometry (VBM) pipeline in SPM12, which has been described in detail [23]. Briefly, each AC-PC aligned T1-weighted image was segmented into grey matter, white matter, and CSF tissue classes using SPM12's unified segmentation procedure, followed by the creation of a study-specific DARTEL template [24-26]. Segmented images were then iteratively aligned to the DARTEL template, spatially normalized, modulated, and smoothed with an $8 \mathrm{~mm}$ full-width at half-maximum isotropic Gaussian kernel. Resulting smoothed, modulated, and warped tissue segmentations were used in subsequent analyses.

Region-of-interest (ROI) masks extracted whole brainstem, midbrain, pons, and locus coeruleus (LC) volumes (Supplementary Fig. 1). A previously established ROI mask defined by the grey and white matter tissue maps from the ICBM152 template was used to assess whole brainstem volumes comprising the pons, medulla, and midbrain (Supplementary Fig. 1A) [27-30]. ROI masks for the midbrain and pons were obtained from an atlas created as part of a study establishing a probabilistic Bayesian segmentation procedure for automated delineation of the brainstem and its sub-regions, and these masks have been validated in clinical populations (e.g., progressive supranuclear palsy, corticobasal syndrome) known to experience atrophy of these regions (Supplementary Fig. 1B-C) [31,32]. To approximate LC volume, we used a previously created ROI mask derived by averaging coordinates for peak voxels of functional activity and neuromelanin sensitivity from two prior studies that localized the LC on functional MRI and T1-weighted turbo spin echo MRI scans (Supplementary Fig. 1D) (https://rcweb.dartmouth.edu/CANlab/brainstemwiki/doku.php/ lc.html) [33,34]. Volumes for brainstem ROIs were calculated by summing grey and white matter voxel values from the VBM-processed images; both grey and white matter were included for the brainstem due to the mixed tissue classifications that make up the structure [35]. Total intracranial volume (TIV) was calculated as the sum of all voxels across the grey 
matter, white matter, and CSF segmented maps. Volume extractions for TIV and all ROIs were performed using built-in SPM12 functions (e.g. "spm_summarise") and the "get_totals" script (http://www0.cs.ucl.ac.uk/staff/gridgway/vbm/get_totals.m). To correct for head size, ROI volumes were normalized via simple division by TIV, a widely used method for volumetric normalization [14,36,37]. Additional volume normalizations for midbrain, pons, and LC ROIs were performed via division by whole brainstem volume to determine specificity of observed volumetric differences. Normalized volumes were subsequently multiplied by a factor of $10^{3}$ (whole brainstem, midbrain, pons) or $10^{4}$ (LC) to facilitate ease of comparisons.

In addition to ROI analyses, voxel-wise two sample t-tests examined morphometric differences between groups. White matter maps were selected due to SPM's predominant classification of the brainstem as white matter, and prior work has demonstrated the feasibility of detecting brainstem abnormalities with VBM in clinical populations [15,16,32]. VBM maps were statistically compared between groups using two-sample t-tests with age, sex, education, $A P O E \varepsilon 4$ status, and TIV included as covariates. Additional models replaced the TIV covariate with TIV-normalized pons volume or TIV-normalized brainstem volume to address potential concerns of regional specificity. An explicit mask combining the midbrain and pons restricted analyses to rostral brainstem structures. A height threshold of $p<.05$ with family-wise error (FWE) correction for multiple comparisons was used and resulting maps were inspected for significant clusters representing groupwise volumetric differences. Where noted below, less stringent height thresholds of uncorrected $p$ $<.05$ and $p<.01$ were used for exploratory analyses in cases where no differences were observed at FWE-corrected $p<.05$. VBM analyses listed below compared clinicallydiagnosed groups, rather than biomarker-confirmed groups, to preserve subject numbers and ensure sufficient statistical power.

\section{CSF Biomarkers}

A subset of ADNI participants underwent fasting lumbar puncture at baseline, and levels of amyloid $\beta$ 1-42 (A $\left.\beta_{1-42}\right)$ and phosphorylated tau (pTau) were quantified using the automated Roche Elecsys $A \beta_{1-42}$ CSF and Elecsys phosphotau (181P) CSF electrochemiluminescene immunoassays at the UPenn Biomarker Research Laboratory [38]. Participants were categorized as amyloid-positive with values of $A \beta_{1-42}$ below $980 \mathrm{pg} / \mathrm{ml}$ and as amyloid-negative with values at $980 \mathrm{pg} / \mathrm{ml}$ and above. They were also categorized as pTau-positive with values of $\mathrm{pTau}_{181 \mathrm{p}}$ at or above $21.8 \mathrm{pg} / \mathrm{ml}$ and as pTau-negative with values below $21.8 \mathrm{pg} / \mathrm{ml}$ (see http://adni.loni.usc.edu/methods/ for more information) [39]. Participants who were both amyloid-positive and pTau-positive were considered biomarkerconfirmed AD or MCI due to AD (MCI n= 203; AD n = 160) [39,40].

\section{Statistical Analyses}

All continuous variables were checked for normality via skewness and kurtosis. Substantial departure from normality was noted for the Trails A, Trails B, and Boston Naming Test variables, and a $\log _{10}$ transformation was applied. The Boston Naming Test variable was reflected prior to $\log _{10}$ transformation to avoid undefined values. ANOVA with Tukey's post-hoc test was used to test group differences in age and education. $\chi^{2}$ test was used to test 
group differences in sex and $A P O E \varepsilon 4$ status. ANCOVA with age, sex, education, and $A P O E \varepsilon 4$ status as covariates tested group differences in neuroimaging variables, with posthoc LSD tests used for pairwise group comparisons.

Longitudinal analyses were restricted to cognitively normal participants with at least one follow-up timepoint of clinical diagnostic data. Proportional hazards survival analyses were conducted via Cox regressions to determine the value of brainstem ROI volumes in predicting progression from normal cognition to clinically-diagnosed AD dementia. In Cox regression models, continuous brainstem ROI volume was first entered as a sole predictor variable, with $\mathrm{AD}$ dementia diagnosis as event of interest and months to diagnosis as time variable. Next, for models that were significant with a single predictor variable, relevant covariates of age, sex, education, and $A P O E \varepsilon 4$ status were added in a second block. Hazard ratios (HR) and 95\% confidence intervals (CI) were reported from all Cox regression models with brainstem ROI volumes entered as continuous predictor variables. Median splits were performed solely for visualization of risk profiles at high versus low volumes, with survival curves plotted for groups split by high or low brainstem ROI volume. To address potential bias introduced by selective attrition, all Cox regression models were initially run with variable follow-up (6-120 months) and repeated with a fixed 48-month follow-up. Additional Cox regression analyses assessed value of brainstem ROI volumes in predicting progression from ADNI-diagnosed normal cognition to ADNI-diagnosed MCI.

To address the issue of multiple comparisons, false discovery rate (FDR) correction via the Benjamini-Hochberg procedure was applied to omnibus tests for all planned comparisons, including diagnostic group and biomarker-split group comparisons of ROI volumes, posthoc pairwise tests, and Cox regressions of converters/non-converters to dementia [41]. Results were considered significant at FDR-corrected threshold of .05 and .10. For all other unplanned comparisons (e.g., demographic variables), results were considered significant at Bonferroni adjusted $p<.05$. All statistical analyses were performed in SPSS (IBM SPSS Statistics 25, IBM, Armonk, NY, USA) and Prism (GraphPad Prism 7, GraphPad Software Inc., San Diego, CA, USA).

\section{RESULTS \\ Demographics}

Baseline demographic data are displayed for clinically-diagnosed and biomarker-confirmed groups in Table 1 and for AD dementia converters and non-converters in Table 2.

\section{Brainstem Region-of-interest Analyses}

Relative to the cognitively normal group, participants with neuropsychologically-confirmed MCI or clinically-diagnosed AD dementia had smaller TIV-normalized whole brainstem $\left[F(2,1615)=7.13, p=.001, \eta_{p}{ }^{2}=.009\right]$, midbrain $\left[F(2,1617)=16.80, p<.001, \eta_{p}{ }^{2}=.02\right]$, and pons $\left[F(2,1616)=3.26, p=.039, \eta_{p}{ }^{2}=.004\right]$ volumes (Table 1; Fig. $1 \mathrm{~A}-\mathrm{C}$; Supplementary Table 1). Diagnostic groups did not differ in TIV. When normalizing brainstem substructures to whole brainstem volume, participants with AD dementia had smaller midbrain relative to whole brainstem volume $\left[F(2,1617)=5.70, p=.003, \eta_{p}{ }^{2}\right.$ 
$=.007]$ and larger pons relative to whole brainstem volume $\left[F(2,1620)=7.12, p=.001 \eta_{p}{ }^{2}\right.$

$=.009 \mathrm{]}$ as compared to the cognitively normal group (Supplementary Table 2A,D). When constraining analyses to biomarker-confirmed groups, AD dementia participants exhibited smaller TIV-normalized midbrain $\left[F(2,1150)=4.58, p=.01, \eta_{p}{ }^{2}=.008\right]$ volumes than cognitively normal participants, and there were no differences in whole brainstem or pons volumes (all p's > .10) (Table 1; Fig. 1D-F; Supplementary Table 1). When normalizing brainstem substructures to whole brainstem volume, biomarker-confirmed AD dementia participants had smaller midbrain relative to whole brainstem volume $[F(2,1150)=5.07, p$ $\left.=.006, \eta_{p}{ }^{2}=.009\right]$ as compared to the cognitively normal group and larger pons relative to whole brainstem volume $\left[F(2,1152)=7.84, p<.001, \eta_{p}{ }^{2}=.013\right]$ as compared to the cognitively normal and MCI groups (Supplementary Table 2B,D). Cognitively normal participants who progressed to AD dementia (converters) had smaller baseline TIVnormalized midbrain $\left[F(1,775)=8.68, p=.003, \eta_{p}{ }^{2}=.011\right]$ volumes than those who did not progress to dementia (non-converters), and no differences were observed in TIV, TIVnormalized whole brainstem volume, TIV-normalized pons volume, or brainstemnormalized ROIs (Table 2; Fig. 2A-C; Supplementary Table 2C; see Supplementary Table 3 for life table). With FDR limited to 0.05 , Cox regression analyses of relationships between baseline TIV-normalized ROI volumes and progression to dementia demonstrated that smaller baseline midbrain volume was associated with higher risk of progression to dementia (HR 3.24, 95\% CI [1.51, 6.96], $p=.003$ ) (Fig. 2E). With FDR limited to 0.10, smaller baseline whole brainstem volume was associated with higher risk of progression to dementia (HR $=1.24,95 \%$ CI [1.02, 1.52], $p=.033$ ) (Fig. 2D). Cox regression with baseline pons volume as predictor was not significant, nor were repeated Cox regression models with baseline brainstem-normalized ROI volumes as predictor variables (Fig. 2F; Supplementary Table 2E). Repeated Cox regression models with fixed 48-month follow-up periods confirmed that smaller baseline midbrain volume was associated with higher risk of progression to dementia (HR 3.14, 95\% CI [1.47, 6.69], $p=.003$ ], however relationships for brainstem and pons were not significant (Supplementary Table 4A-B).

ROI analyses centered on the LC indicated that participants with neuropsychologicallyconfirmed MCI or clinically-diagnosed AD dementia had smaller TIV-normalized LC $\left[F(2,1616)=4.50, p=.011, \eta_{p}{ }^{2}=.006\right]$ volumes relative to the cognitively normal group, while no differences were observed when constraining analyses to biomarker-confirmed groups or when normalizing to whole brainstem volume (Table 1; Fig. 3C-D;

Supplementary Table 1; Supplementary Table 2A). Baseline LC ROI volumes did not differ between AD dementia converters and non-converters at baseline when normalizing to TIV or to whole brainstem volume (Fig. 4A; Table 2; Supplementary Table 2C). With FDR limited to 0.10 , Cox regression analyses demonstrated that smaller baseline LC volume conferred higher risk of progression to dementia (HR 9.10, 95\% CI [1.20, 69.22], $p=.033$ ) (Fig. 4B). Baseline LC volume was not predictive of progression to dementia in repeated Cox regression models with fixed 48-month follow-up period or in models with brainstemnormalized LC volume (Supplementary Table 2E; Supplementary Table 4).

ROI analyses were additionally repeated with raw ROI volumes (as opposed to TIVnormalized volumes) and TIV included as a covariate in statistical models; this approach did not affect any results. In a risk analysis examining ADNI-diagnosed $\mathrm{CN}$ individuals who 
progressed to an ADNI diagnosis of MCI, baseline midbrain volume was associated with higher risk of MCI diagnosis (HR 2.26, 95\% CI [1.20, 4.27], $p=.012$ ) (Supplementary Table 5A-B).

\section{Brainstem-Masked VBM Analyses}

Brainstem-masked VBM analyses of the entire MCI sample, regardless of biomarker positivity, compared to cognitively normal participants indicated smaller regional volume of clusters overlapping the bilateral LC and bilateral clusters in the anterolateral midbrain (Fig. 3A; Supplementary Table 6A). Patterns of regional volume difference between AD dementia and cognitively normal participants similarly indicated smaller clusters overlapping bilateral LC, anterolateral midbrain, and dorsal rostral pontine regions (Fig. 3B; Supplementary Table 6B). All VBM findings were significant at an FWE-corrected height threshold of $p<.05$. Brainstem-masked VBM analyses within cognitively normal participants showed smaller regional volume of bilateral clusters corresponding to the anatomical distribution of the LC in $\mathrm{AD}$ dementia converters compared to non-converters, the only clusters that remained significant at an uncorrected height threshold of $p<.01$ (Fig. 4C; Supplementary Table 7A). When observed at a less stringent threshold of uncorrected $p<.05$, the clusters of interest extended caudally, further overlapping the anatomical distribution of the LC (Supplementary Table 7B; Supplementary Fig. 2). We compared MNI coordinates from our voxel-wise analyses and found specific overlap with prior VBM studies of the brainstem and with studies that have localized the structure of the LC on neuromelanin-sensitive T1-weighted sequences (Supplementary Table 8). In order to demonstrate that voxel-wise findings were not a reflection of overall pons or overall brainstem volume difference, VBM analyses were repeated with covariates for TIV-normalized pons volume and TIV-normalized whole brainstem volume in place of the TIV covariate. Clusters overlapping the LC remained significant at FWE-corrected $p<.05$ in $\mathrm{AD}$ compared to $\mathrm{CN}$ with pons covariate, and at uncorrected $p<.05$ in AD compared to $\mathrm{CN}$ with whole brainstem covariate, as well as MCI compared to $\mathrm{CN}$ with pons covariate and whole brainstem covariate (Supplementary Figs. 3-4). Similarly, clusters overlapping the LC remained significant at uncorrected $p<.05$ in $\mathrm{AD}$ dementia converters compared to non-converters when controlling for TIV-normalized pons and whole brainstem volumes (Supplementary Figs. 5-6).

\section{DISCUSSION}

The current study found that older adults with biomarker-confirmed dementia due to AD exhibited smaller midbrain volumes than cognitively normal individuals. Furthermore, smaller midbrain volumes were observed in cognitively normal older adults who later went on to develop AD dementia compared to those who did not progress to dementia, and lower baseline brainstem, midbrain, and LC volumes were predictive of future progression to $\mathrm{AD}$ dementia diagnosis. These findings confirm prior MRI studies implicating brainstem volumetric differences in clinically-diagnosed AD dementia and further clarify that these differences are observable earlier in AD pathophysiological processes [12,15,31]. A brainstem-masked analysis using voxel-level methods revealed further brainstem differences between neuropsychologically-confirmed $\mathrm{MCI}$ and clinically-diagnosed dementia due to $\mathrm{AD}$ compared to cognitively normal individuals in a small cluster corresponding with the 
anatomical location of the $\mathrm{LC}$ along the midbrain-pontine axis. Our findings provide preliminary in vivo evidence of structural brainstem abnormalities detectible on traditional MRI sequences, mirroring neuropathological studies that have localized early AD pathology to brainstem nuclei $[42,43]$. Taken together, the patterns of brainstem volumetric differences across clinically-diagnosed and biomarker-confirmed $\mathrm{AD}$ groups suggest early brainstem pathology in the midbrain and LC is observable on MRI, and this pathology is predictive of clinical progression from the earliest preclinical phase of the disease.

A strength of the present study was the use of VBM in addition to ROI-based volumetrics to compare diagnostic groups and constrain analyses to brainstem substructures. Our finding of reduced LC volume in $\mathrm{MCI}$ and $\mathrm{AD}$ dementia compared to cognitively normal individuals provides a volumetric analogue to previous studies showing reduced LC neuromelanin contrast ratios in $\mathrm{AD}$ and $\mathrm{MCI}$ relative to cognitively normal individuals [44,45]. To our knowledge, no prior studies have outlined proxy estimates of LC volume on traditional T1weighted images and instead have focused on neuromelanin-sensitive T1-weighted scans. Our study represents the first known effort to evaluate LC integrity with volumetrics as opposed to neuromelanin contrast ratio. Despite the difficulty in quantifying the volume of a nucleus as small as the LC on structural MRI, groupwise differences in LC volume were in the expected direction, with smaller LC volume seen in cognitive impairment and predicting future cognitive impairment. Clusters corresponding to the bilateral LC resulting from our voxel-wise analyses overlap with coordinates reported from prior studies using neuromelanin-sensitive T1-weighted FSE scans, demonstrating the potential utility of traditional T1-weighted scans in detecting LC volumetric differences [34,46,47]. Our analyses indicated gross detectible differences in the midbrain while voxel-level analyses revealed sub-regional differences in midbrain and pontine regions adjacent to nuclei known to degenerate with advancing Braak stage [43]. Our midbrain-specific findings in biomarkerconfirmed $\mathrm{AD}$ cases emphasize that volumetric differences are detectible in a structure known to undergo selective neuronal loss in the earliest precortical stages of $\mathrm{AD}$ and may be indicative of compromised optimal regulation of various arousal-related systems (e.g., serotonergic, glutamatergic, cholinergic, noradrenergic) in AD progression. [1,43,48]. Future ex vivo neuropathological studies will be helpful in clarifying whether our findings represent a proxy measure of neurodegeneration, synaptic loss, axonal deterioration, or a different neuropathological marker, but in any case our approach has established detectible differences in brainstem MRI in very early stage AD.

Given that smaller midbrain volume in cognitively normal older adults predicts future dementia, it is possible that greater premorbid midbrain volume confers a degree of protection against insidious tau deposition and consequent disease progression. This is consistent with the neural reserve literature suggesting the $\mathrm{LC}$ as a site of reserve due to its known involvement in higher-order executive cognitive processes, neuroplasticity, memory, and arousal, and our findings support a critical role of the LC in protecting against the deleterious effects and corresponding clinical consequences of increasing AD pathology [49-51]. Alternatively, our findings could be interpreted as suggesting brainstem regions are selectively vulnerable to neurodegenerative disease. As we demonstrated, reduced premorbid structural integrity of the midbrain and LC bestows greater risk for cognitive decline and disease progression, in line with studies observing reduced LC neuromelanin 
contrast ratios in $\mathrm{AD}$ dementia and $\mathrm{MCI}$ populations compared to cognitively normal individuals, inverse correlations between $\mathrm{LC}$ neuromelanin contrast ratio and $\mathrm{A} \beta$ pathological burden, and general declines in LC neuromelanin contrast with age $[44,45,52,53]$. Prior studies have examined LC degeneration in the early stages of AD pathophysiology in postmortem tissue, and the present study provides preliminary evidence that these differences may be apparent in vivo with widely used structural neuroimaging techniques $[1,2,54,55]$. The LC's role as the primary site of noradrenergic production and regulation has increased its potential as a biomarker for neurodegenerative disease, and our findings suggest that gross anatomical differences quantified on T1-weighted scans may be useful when assessing preclinical and prodromal populations in contexts where more advanced imaging sequences are unavailable [56,57].

Alternative pathways of dysfunction must also be considered when examining a nucleus as functionally diverse as the LC. Recent animal studies have suggested that LC integrity is linked to optimal regulation of cerebral blood flow, with degeneration of the LCnorepinephrine projection system predicting downstream vascular consequences in ADrelated regions [58,59]. Multimodal neuroimaging and biomarker studies of wellcharacterized clinical populations are needed to disentangle whether subcortical brainstem nuclei represent sites of selective vulnerability or resilience to AD pathological burden. In the context of ongoing debate over whether subcortical regional pathology in the LC and other brainstem nuclei represent the initial site of tau seeding and hyperphosphorylated tau deposition in $\mathrm{AD}$, our study cannot argue one way or the other due to the lack of histopathological analysis and Braak staging. However, the observation of early brainstem volume differences in $\mathrm{MCI}$ due to $\mathrm{AD}$ and cognitively normal older adults at risk for future $\mathrm{AD}$ dementia diagnosis clearly emphasizes the importance of integrating in vivo neuroimaging studies with histopathological studies to continue characterizing the diseaserelated progression of tau pathology.

The present study is not without several caveats. One limitation is the estimation of LC volume from structural T1 images via approximated ROI masks. The LC is a notoriously difficult nucleus to localize on images and neuromelanin-sensitive T1-weighted fast spin echo or turbo spin echo sequences are thought to best visualize and capture the integrity of the LC $[34,47,49]$. These specialized scans leverage the natural accumulation of neuromelanin in noradrenergic cells of the LC over the lifespan, which has paramagnetic T1-shortening effects, and apply targeted scan parameters to capture this neuromelanin concentration as hyperintensities visible in the pons $[46,60]$. The present study used T1weighted volumetric sequences and an LC mask centered on average peak voxels from multiple studies to approximate LC volume. Future studies will need to compare this methodology to neuromelanin-sensitive sequences [33,34]. Although we demonstrated groupwise differences among diagnostic groups in ROI volume of LC when normalizing to TIV, this finding did not remain significant when normalizing to whole brainstem volume. This may represent artifact specific to our methodology, as various processing steps including warping to template space and spatial smoothing make it difficult to ensure regional specificity in a mixed tissues structure such as the brainstem. VBM methodologies contain inherent limitations for evaluating grey/white matter contrast in a mixed tissue class structure such as the brainstem, and it has been previously noted that VBM may have a 
limited capacity to detect subtle changes in white matter regions that are largely homogenous in nature [61,62]. However, prior studies have used VBM analysis of white matter maps to successfully detect volume loss within brainstem substructures in disease populations known to experiences specific volume loss within the brainstem (progressive supranuclear palsy and corticobasal degeneration), as well as in Alzheimer's disease compared to healthy controls $[15,32]$. Our study further adds to this literature by providing additional support for the feasibility of this approach within the greater context of the limitations VBM analyses pose. Additionally, regional findings from our voxel-wise analyses remained significant when correcting for total pons volume or total brainstem volume. Nevertheless, future studies should conduct similar analyses in cohorts with specialized neuromelanin-sensitive MRI sequences designed to specifically assess LC structural integrity [57].

Survival analyses indicated that baseline LC volume confers significant risk for dementia, but the large 95\% CI suggests the stability of this prediction is highly variable, and predictive value was no longer significant when examining over a fixed 48-month follow-up. Additionally, the present study analyzed a sample with high rates of MCI and AD cases likely not present in real-world settings, suggesting that a similar analysis in a smaller sample would result in lower predictive value. Thus, the predictive value of the LC should be interpreted with caution and future studies in more representative samples will help to determine the stability of predictive values and may aim to make comparisons with regions well-established to experience atrophic changes in AD dementia (e.g., hippocampus, medial temporal lobe). Nevertheless, the close anatomical localization provided from the LC ROI mask, combined with the marked volumetric differences observed in VBM analyses, implicate midbrain and pontine regions adjacent to and overlapping the LC in preclinical AD. Given the clinical subjectivity of differential diagnoses in ADNI and the lack of pathological diagnostic confirmation in our cohort, it is possible that our findings in the clinically-defined groups were influenced by individuals with co-morbid subcortical dementias, primary age-related tauopathies, and other non-AD processes. We examined cross-sectional volumetric differences at baseline rather than progression of brain atrophy over time purposefully in an effort to comprehensively quantify baseline volumetrics. Future studies will aim to detail the longitudinal progression of brainstem substructure atrophy in each of our diagnostic groups. It should be noted that although our analysis predicting AD dementia used neuropsychologically-confirmed cluster analysis to refine baseline MCI diagnoses, our analysis predicting MCI used original ADNI MCI diagnoses. Future efforts should use neuropsychological data to inform serial MCI diagnoses to avoid potential misclassifications, as demonstrated by recent studies [63]. Additionally, our longitudinal risk analyses included clinically-diagnosed groups and were not performed in biomarkerconfirmed groups due to limited sample sizes. Thus, longitudinal studies are needed to detail brainstem atrophy progression in biomarker-confirmed MCI and AD. Finally, the ADNI database comprises an ethnically homogenous, highly educated sample that is not necessarily representative of the general population, and future studies will need to replicate our findings in diverse cohorts.

Despite these limitations, our study demonstrates brainstem MRI abnormalities that are detectible in preclinical populations, highlighting the importance of considering the 
brainstem when developing novel biomarkers and innovative therapeutics. Future studies should leverage additional neuroimaging modalities, including functional MRI, diffusion tensor imaging, and arterial spin labeling, to address issues of neurovascular coupling, structural white matter tract degeneration, and cerebral perfusion as they relate to the brainstem and disease progression. Future endeavors should also separate individuals by Braak stage and quantify these brainstem substructure volumes to examine whether brainstem atrophy progresses in temporal conjunction with the widely accepted pathophysiological staging advancement. Future MRI-based studies of dementia populations may aim to include brainstem volumetrics as outcome variables and address their potential utility as clinical trial endpoints $[15,31,64]$. In summary, we provide here preliminary evidence that in vivo visualization of brainstem substructure and LC-specific differences are detectible with widely used MRI sequences in preclinical and prodromal AD populations.

\title{
Supplementary Material
}

Refer to Web version on PubMed Central for supplementary material.

\section{ACKNOWLEDGMENTS}

\begin{abstract}
We would like to thank the participants and their families, investigators, and researchers from the ADNI study. Data used in preparation of this article were obtained from the ADNI database (adni.loni.usc.edu). As such, the investigators within the ADNI contributed to the design and implementation of ADNI and/or provided data but did not participate in analysis or writing of this report. A complete listing of ADNI investigators can be found at: http:// adni.loni.usc.edu/wp-content/uploads/how_to_apply/ADNI_Acknowledgement_List.pdf

Author funding for this study was obtained through grants from the National Institutes of Health (R01AG060049, R21AG055034, P01AG052350, P50AG00514, P50AG016573, R01AG025340, R01AG64228), the Alzheimer's Association (AA008369), and the National Science Foundation (DGE1418060). Data collection and sharing for this project was funded by the Alzheimer's Disease Neuroimaging Initiative (ADNI) (National Institutes of Health Grant U01 AG024904) and DOD ADNI (Department of Defense award number W81XWH-12-2-0012). ADNI is funded by the National Institute on Aging, the National Institute of Biomedical Imaging and Bioengineering, and through generous contributions from the following: AbbVie, Alzheimer's Association; Alzheimer's Drug Discovery Foundation; Araclon Biotech; BioClinica, Inc.; Biogen; Bristol-Myers Squibb Company; CereSpir, Inc.; Cogstate; Eisai Inc.; Elan Pharmaceuticals, Inc.; Eli Lilly and Company; EuroImmun; F. Hoffmann-La Roche Ltd and its affiliated company Genentech, Inc.; Fujirebio; GE Healthcare; IXICO Ltd.; Janssen Alzheimer Immunotherapy Research \& Development, LLC.; Johnson \& Johnson Pharmaceutical Research \& Development LLC.; Lumosity; Lundbeck; Merck \& Co., Inc.; Meso Scale Diagnostics, LLC.; NeuroRx Research; Neurotrack Technologies; Novartis Pharmaceuticals Corporation; Pfizer Inc.; Piramal Imaging; Servier; Takeda Pharmaceutical Company; and Transition Therapeutics. The Canadian Institutes of Health Research is providing funds to support ADNI clinical sites in Canada. Private sector contributions are facilitated by the Foundation for the National Institutes of Health (www.fnih.org). The grantee organization is the Northern California Institute for Research and Education, and the study is coordinated by the Alzheimer's Therapeutic Research Institute at the University of Southern California. ADNI data are disseminated by the Laboratory for NeuroImaging at the University of Southern California.
\end{abstract}

\section{REFERENCES}

[1]. Grinberg LT, Rüb U, Ferretti REL, Nitrini R, Farfel JM, Polichiso L, Gierga K, Jacob-Filho W, Heinsen H (2009) The dorsal raphe nucleus shows phospho-tau neurofibrillary changes before the transentorhinal region in Alzheimers disease. A precocious onset? Neuropathol Appl Neurobiol 35, 405-416.

[2]. Simic G, Stanic G, Mladinov M, Jovanov-Milosevic N, Kostovic I, Hof PR (2009) Does Alzheimer's disease begin in the brainstem? Neuropathol Appl Neurobiol 35, 532-554. [PubMed: 19682326]

[3]. Braak H, Del Tredici K (2015) The preclinical phase of the pathological process underlying sporadic Alzheimer's disease. Brain 138, 2814-2833. [PubMed: 26283673] 
[4]. Braak H, Thal DR, Ghebremedhin E, Del Tredici K (2011) Stages of the Pathologic Process in Alzheimer Disease. J Neuropathol Exp Neurol 70, 960-969. [PubMed: 22002422]

[5]. Braak H, Tredici K Del (2018) Spreading of tau pathology in sporadic Alzheimer's disease along cortico-cortical top-down connections. Cereb Cortex 28, 3372-3384. [PubMed: 29982389]

[6]. Kaufman SK, Del Tredici K, Thomas TL, Braak H, Diamond MI (2018) Tau seeding activity begins in the transentorhinal/entorhinal regions and anticipates phospho-tau pathology in Alzheimer's disease and PART. Acta Neuropathol 136, 57-67. [PubMed: 29752551]

[7]. Kaufman SK, Del Tredici K, Braak H, Diamond MI (2018) Rebuttal to Drs. Grinberg and Heinsen. Acta Neuropathol 136, 819-819. [PubMed: 30284035]

[8]. Heinsen H, Grinberg LT (2018) On the origin of tau seeding activity in Alzheimer's disease. Acta Neuropathol 136, 815-817. [PubMed: 30039213]

[9]. Weinshenker D (2008) Functional Consequences of Locus Coeruleus Degeneration in Alzheimers Disease. Curr Alzheimer Res 5, 342-345. [PubMed: 18537547]

[10]. Rüb U, Stratmann K, Heinsen H, Del Turco D, Seidel K, den Dunnen W, Korf H-W (2016) The brainstem tau cytoskeletal pathology of Alzheimer's disease: A brief historical overview and description of its anatomical distribution pattern, evolutional features, pathogenetic and clinical relevance. Curr Alzheimer Res 13, 1178-1197. [PubMed: 27264543]

[11]. Uematsu M, Nakamura A, Ebashi M, Hirokawa K, Takahashi R, Uchihara T (2018) Brainstem tau pathology in Alzheimer's disease is characterized by increase of three repeat tau and independent of amyloid $\beta$. Acta Neuropathol Commun 6, 1-18. [PubMed: 29298724]

[12]. Lee JH, Ryan J, Andreescu C, Aizenstein H, Lim HK (2015) Brainstem morphological changes in Alzheimer's disease. Neuroreport 26, 411-415. [PubMed: 25830491]

[13]. Iglesias JE, Van Leemput K, Bhatt P, Casillas C, Dutt S, Schuff N, Truran-Sacrey D, Boxer A, Fischl B (2015) Bayesian segmentation of brainstem structures in MRI. Neuroimage 113, 184 195. [PubMed: 25776214]

[14]. Mrzilková J, Zach P, Bartoš A, Tintěra J, Řípová D (2012) Volumetric analysis of the pons, cerebellum and hippocampi in patients with Alzheimer's disease. Dement Geriatr Cogn Disord 34, 224-234. [PubMed: 23128238]

[15]. Nigro S, Cerasa A, Zito G, Perrotta P, Chiaravalloti F, Donzuso G, Fera F, Bilotta E, Pantano P, Quattrone A (2014) Fully automated segmentation of the pons and midbrain using human T1 MR brain images. PLoS One 9, e85618. [PubMed: 24489664]

[16]. Ji X, Wang H, Zhu M, He Y, Zhang H, Chen X, Gao W, Fu Y (2020) Brainstem atrophy in the early stage of Alzheimer's disease: a voxel-based morphometry study. Brain Imaging Behav.

[17]. Petersen RC, Aisen PS, Beckett LA, Donohue MC, Gamst AC, Harvey DJ, Jack CR, Jagust WJ, Shaw LM, Toga AW, Trojanowski JQ, Weiner MW (2010) Alzheimer's Disease Neuroimaging Initiative (ADNI): Clinical characterization. Neurology 74, 201-209. [PubMed: 20042704]

[18]. Edmonds EC, Delano-Wood L, Clark LR, Jak AJ, Nation DA, McDonald CR, Libon DJ, Au R, Galasko D, Salmon DP, Bondi MW (2015) Susceptibility of the conventional criteria for mild cognitive impairment to false-positive diagnostic errors. Alzheimer's Dement 11, 415-424. [PubMed: 24857234]

[19]. Delano-Wood L, Bondi MW, Sacco J, Abeles N, Jak AJ, Libon DJ, Bozoki A (2009) Heterogeneity in mild cognitive impairment: Differences in neuropsychological profile and associated white matter lesion pathology. J Int Neuropsychol Soc 15, 906-914. [PubMed: 19891820]

[20]. Clark LR, Delano-Wood L, Libon DJ, Mcdonald CR, Nation DA, Bangen KJ, Jak AJ, Au R, Salmon DP, Bondi MW (2013) Are empirically-derived subtypes of mild cognitive impairment consistent with conventional subtypes? J Int Neuropsychol Soc 19, 635-645. [PubMed: 23552486]

[21]. Marchewka A, Kherif F, Krueger G, Grabowska A, Frackowiak R, Draganski B (2014) Influence of magnetic field strength and image registration strategy on voxel-based morphometry in a study of Alzheimer's disease. Hum Brain Mapp 35, 1865-1874. [PubMed: 23723177]

[22]. Jack CR, Barnes J, Bernstein MA, Borowski BJ, Brewer J, Clegg S, Dale AM, Carmichael O, Ching C, DeCarli C, Desikan RS, Fennema-Notestine C, Fjell AM, Fletcher E, Fox NC, Gunter J, Gutman BA, Holland D, Hua X, Insel P, Kantarci K, Killiany RJ, Krueger G, Leung KK, 
Mackin S, Maillard P, Malone IB, Mattsson N, McEvoy L, Modat M, Mueller S, Nosheny R, Ourselin S, Schuff N, Senjem ML, Simonson A, Thompson PM, Rettmann D, Vemuri P, Walhovd K, Zhao Y, Zuk S, Weiner M (2015) Magnetic resonance imaging in Alzheimer's Disease Neuroimaging Initiative 2. Alzheimer's Dement 11, 740-756. [PubMed: 26194310]

[23]. Ashburner J, Friston KJ (2000) Voxel-Based Morphometry-The Methods. Neuroimage 11, 805821. [PubMed: 10860804]

[24]. Ashburner J, Friston KJ (2005) Unified segmentation. Neuroimage 26, 839-851. [PubMed: 15955494]

[25]. Ashburner J (2007) A fast diffeomorphic image registration algorithm. Neuroimage 38, 95-113. [PubMed: 17761438]

[26]. Ashburner J, Friston KJ (2009) Computing average shaped tissue probability templates. Neuroimage 45, 333-341. [PubMed: 19146961]

[27]. Beissner F, Schumann A, Brunn F, Eisenträger D, Bär KJ (2014) Advances in functional magnetic resonance imaging of the human brainstem. Neuroimage 86, 91-98. [PubMed: 23933038]

[28]. Mazziotta J, Toga A, Evans A, Fox P, Lancaster J, Zilles K, Woods R, Paus T, Simpson G, Pike B, others (2001) A four-dimensional probabilistic atlas of the human brain. J Am Med Informatics Assoc 8, 401-430.

[29]. Mazziotta J, Toga A, Evans A, Fox P, Lancaster J, Zilles K, Woods R, Paus T, Simpson G, Pike B, Holmes C, Collins L, Thompson P, MacDonald D, Iacoboni M, Schormann T, Amunts K, Palomero-Gallagher N, Geyer S, Parsons L, Narr K, Kabani N, Goualher GL, Boomsma D, Cannon T, Kawashima R, Mazoyer B (2001) A probabilistic atlas and reference system for the human brain: International Consortium for Brain Mapping (ICBM). Philos Trans R Soc B Biol Sci 356, 1293-1322.

[30]. Mazziotta JC, Toga AW, Evans A, Fox P, Lancaster J (1995) A probabilistic atlas of the human brain: theory and rationale for its development. The International Consortium for Brain Mapping (ICBM). Neuroimage 2, 89-101. [PubMed: 9343592]

[31]. Iglesias JE, Van Leemput K, Bhatt P, Casillas C, Dutt S, Schuff N, Truran-Sacrey D, Boxer A, Fischl B (2015) Bayesian segmentation of brainstem structures in MRI. Neuroimage 113, 184195. [PubMed: 25776214]

[32]. Dutt S, Binney RJ, Heuer HW, Luong P, Attygalle S, Bhatt P, Marx GA, Elofson J, Tartaglia MC, Litvan I, McGinnis SM, Dickerson BC, Kornak J, Waltzman D, Voltarelli L, Schuff N, Rabinovici GD, Kramer JH, Jack CR, Miller BL, Rosen HJ, Boxer AL (2016) Progression of brain atrophy in PSP and CBS over 6 months and 1 year. Neurology 87, 2016-2025. [PubMed: 27742814]

[33]. Astafiev SV, Snyder AZ, Shulman GL, Corbetta M (2010) Comment on "Modafinil shifts human locus coeruleus to low-tonic, high-phasic activity during functional MRI" and "Homeostatic sleep pressure and responses to sustained attention in the suprachiasmatic area." Science (80-) $328,$.

[34]. Keren NI, Lozar CT, Harris KC, Morgan PS, Eckert MA (2009) In vivo mapping of the human locus coeruleus. Neuroimage 47, 1261-1267. [PubMed: 19524044]

[35]. Parraga RG, Possatti LL, Alves RV, Ribas GC, Oliveira E De (2016) Microsurgical anatomy and internal architecture of the brainstem in 3D images: surgical considerations. J Neurosurg 124, 1377. [PubMed: 26517774]

[36]. Whitwell JL, Crum WR, Watt HC, Fox NC (2001) Normalization of cerebral volumes by use of intracranial volume: Implications for longitudinal quantitative MR imaging. Am J Neuroradiol 22, 1483-1489. [PubMed: 11559495]

[37]. Nesteruk M, Nesteruk T, Styczyńska M, Mandecka M, Barczak A, Barcikowska M (2016) Combined use of biochemical and volumetric biomarkers to assess the risk of conversion of mild cognitive impairment to Alzheimer's disease. Folia Neuropathol 54, 369-374.

[38]. Bittner T, Zetterberg H, Teunissen CE, Ostlund RE, Militello M, Andreasson U, Hubeek I, Gibson D, Chu DC, Eichenlaub U, Heiss P, Kobold U, Leinenbach A, Madin K, Manuilova E, Rabe C, Blennow K (2016) Technical performance of a novel, fully automated 
electrochemiluminescence immunoassay for the quantitation of $\beta$-amyloid (1-42) in human cerebrospinal fluid. Alzheimer's Dement 12, 517-526. [PubMed: 26555316]

[39]. Hansson O, Seibyl J, Stomrud E, Zetterberg H, Trojanowski JQ, Bittner T, Lifke V, Corradini V, Eichenlaub U, Batrla R, Buck K, Zink K, Rabe C, Blennow K, Shaw LM (2018) CSF biomarkers of Alzheimer's disease concord with amyloid- $\beta$ PET and predict clinical progression : A study of fully automated immunoassays in BioFINDER and ADNI cohorts. Alzheimer's Dement 14, 1470-1481. [PubMed: 29499171]

[40]. Jack CR, Bennett DA, Blennow K, Carrillo MC, Dunn B, Haeberlein SB, Holtzman DM, Jagust W, Jessen F, Karlawish J, Liu E, Molinuevo JL, Montine T, Phelps C, Rankin KP, Rowe CC, Scheltens P, Siemers E, Snyder HM, Sperling R, Elliott C, Masliah E, Ryan L, Silverberg N (2018) NIA-AA Research Framework: Toward a biological definition of Alzheimer's disease. Alzheimer's Dement 14, 535-562. [PubMed: 29653606]

[41]. Glickman ME, Rao SR, Schultz MR (2014) False discovery rate control is a recommended alternative to Bonferroni-type adjustments in health studies. J Clin Epidemiol 67, 850-857. [PubMed: 24831050]

[42]. Braak H, Del Tredici K (2011) The pathological process underlying Alzheimer's disease in individuals under thirty. Acta Neuropathol 121, 171-181. [PubMed: 21170538]

[43]. Stratmann K, Heinsen H, Korf HW, Del Turco D, Ghebremedhin E, Seidel K, Bouzrou M, Grinberg LT, Bohl J, Wharton SB, Den Dunnen W, Rüb U (2016) Precortical Phase of Alzheimer's Disease (AD)-Related Tau Cytoskeletal Pathology. Brain Pathol 26, 371-386. [PubMed: 26193084]

[44]. Takahashi J, Shibata T, Sasaki M, Kudo M, Yanezawa H, Obara S, Kudo K, Ito K, Yamashita F, Terayama Y (2015) Detection of changes in the locus coeruleus in patients with mild cognitive impairment and Alzheimer's disease: High-resolution fast spin-echo T1-weighted imaging. Geriatr Gerontol Int 15, 334-340. [PubMed: 24661561]

[45]. Betts MJ, Cardenas-Blanco A, Kanowski M, Spottke A, Teipel SJ, Kilimann I, Jessen F, Düzel E (2019) Locus coeruleus MRI contrast is reduced in Alzheimer's disease dementia and correlates with CSF A $\beta$ levels. Alzheimer's Dement Diagnosis, Assess Dis Monit 11, 281-285.

[46]. Dahl MJ, Mather M, Düzel S, Bodammer NC, Lindenberger U, Kühn S, Werkle-Bergner M (2019) Rostral locus coeruleus integrity is associated with better memory performance in older adults. Nat Hum Behav 3, 1203-1214. [PubMed: 31501542]

[47]. Betts MJ, Cardenas-Blanco A, Kanowski M, Jessen F, Düzel E (2017) In vivo MRI assessment of the human locus coeruleus along its rostrocaudal extent in young and older adults. Neuroimage 163, 150-159. [PubMed: 28943414]

[48]. Rüb U, Stratmann K, Heinsen H, Seidel K, Bouzrou M, Korf HW (2017) Alzheimer's Disease: Characterization of the Brain Sites of the Initial Tau Cytoskeletal Pathology Will Improve the Success of Novel Immunological Anti-Tau Treatment Approaches. J Alzheimer's Dis 57, 683696. [PubMed: 28269779]

[49]. Clewett DV, Lee TH, Greening S, Ponzio A, Margalit E, Mather M (2015) Neuromelanin marks the spot: Identifying a locus coeruleus biomarker of cognitive reserve in healthy aging. Neurobiol Aging 37, 117-126. [PubMed: 26521135]

[50]. Robertson IH (2013) A noradrenergic theory of cognitive reserve: Implications for Alzheimer's disease. Neurobiol Aging 34, 298-308. [PubMed: 22743090]

[51]. Mather M, Harley CW (2016) The Locus Coeruleus: Essential for Maintaining Cognitive Function and the Aging Brain. Trends Cogn Sci 20, 214-226. [PubMed: 26895736]

[52]. Olivieri $P$, Lagarde J, Lehericy S, Valabrègue R, Michel A, Macé P, Caillé F, Gervais $P$, Bottlaender M, Sarazin M (2019) Early alteration of the locus coeruleus in phenotypic variants of Alzheimer's disease. Ann Clin Transl Neurol 6, 1345-1351. [PubMed: 31353860]

[53]. Liu KY, Acosta-Cabronero J, Cardenas-Blanco A, Loane C, Berry AJ, Betts MJ, Kievit RA, Henson RN, Düzel E, Howard R, Hämmerer D (2019) In vivo visualization of age-related differences in the locus coeruleus. Neurobiol Aging 74, 101-111. [PubMed: 30447418]

[54]. Theofilas P, Ehrenberg AJ, Dunlop S, Alho AT, Nguy A, Elaine Paraizo Leite R, Diehl Rodriguez R, Mejia MB, Suemoto CK, Eloah de Lucena Ferretti-Rebustini R, Polichiso L, Nascimento CF, Seeley WW, Nitrini R, Augusto Pasqualucci C, Jacob Filho W, Rüb U, Neuhaus J, Heinsen H, 
Grinberg LT (2016) Locus coeruleus volume and cell population changes during Alzheimer's disease progression: A stereological study in human postmortem brains with potential implication for early-stage biomarker discovery. Alzheimer's Dement 13, 236-246. [PubMed: 27513978]

[55]. Ehrenberg AJ, Nguy AK, Theofilas P, Dunlop S, Suemoto CK, Alho ATDL, Leite RP, Diehl Rodriguez R, Mejia MB, Rüb U, Farfel JM, de Lucena Ferretti-Rebustini RE, Nascimento CF, Nitrini R, Pasquallucci CA, Jacob-Filho W, Miller B, Seeley WW, Heinsen H, Grinberg LT (2017) Quantifying the accretion of hyperphosphorylated tau in the locus coeruleus and dorsal raphe nucleus: the pathological building blocks of early Alzheimer's Disease. Neuropathol Appl Neurobiol 38, 42-49.

[56]. Dordevic M, Müller-Fotti A, Müller P, Schmicker M, Kaufmann J, Müller NG (2017) Optimal Cut-Off Value for Locus Coeruleus-to-Pons Intensity Ratio as Clinical Biomarker for Alzheimer's Disease: A Pilot Study. J Alzheimer's Dis Reports 1, 159-167.

[57]. Betts MJ, Kirilina E, Otaduy MCG, Ivanov D, Acosta-cabronero J, Callaghan MF, Lambert C, Cardenas-blanco A, Pine K, Passamonti L, Loane C, Mattern H, Liu KY, Priovoulos N, Fliessbach K, Dahl MJ, Maaß A, Madelung CF, Meder D, Ehrenberg AJ, Speck O, Weiskopf N, Dolan R, Inglis B, Tosun D, Morawski M, Zucca FA, Siebner HR, Mather M, Uludag K, Heinsen H, Poser BA, Howard R, Zecca L, Rowe JB, Grinberg LT, Jacobs HIL, Düzel E, Hämmerer D (2019) Locus coeruleus imaging as a biomarker for noradrenergic dysfunction in neurodegenerative diseases. Brain 142, 2558-2571. [PubMed: 31327002]

[58]. Bekar LK, Wei HS, Nedergaard M (2012) The locus coeruleus-norepinephrine network optimizes coupling of cerebral blood volume with oxygen demand. J Cereb Blood Flow Metab 32, 21352145. [PubMed: 22872230]

[59]. Kelly SC, McKay EC, Beck JS, Collier TJ, Dorrance AM, Counts SE (2019) Locus Coeruleus Degeneration Induces Forebrain Vascular Pathology in a Transgenic Rat Model of Alzheimer's Disease. J Alzheimer's Dis 70, 1-18. [PubMed: 31127787]

[60]. Sasaki M, Shibata E, Kudo K, Tohyama K (2008) Neuromelanin-sensitive MRI: Basics, technique, and clinical applications. Clin Neuroradiol 18, 147-153.

[61]. Kurth F, Luders E, Gaser C (2015) Voxel-Based Morphometry. Brain Mapp An Encycl Ref 1, 345-349.

[62]. Mechelli A, Price CJ, Friston KJ, Ashburner J (2005) Voxel-based morphometry of the human brain: methods and applications. Curr Med Imaging Rev 1, 105-113.

[63]. Edmonds EC, Weigand AJ, Hatton SN, Marshall AJ, Thomas KR, Ayala DA, Bondi MW, McDonald CR (2020) Patterns of longitudinal cortical atrophy over 3 years in empirically derived MCI subtypes. Neurology 94, e2532-e2544. [PubMed: 32393648]

[64]. Sander L, Pezold S, Andermatt S, Amann M, Meier D, Wendebourg MJ, Sinnecker T, Yvonne ER, Cristina N, Kappos L, Wuerfel J, Cattin P, Schlaeger R (2019) Accurate, rapid and reliable, fully automated MRI brainstem segmentation for application in multiple sclerosis and neurodegenerative diseases. Hum Brain Mapp 40, 4091-4104. [PubMed: 31206931] 

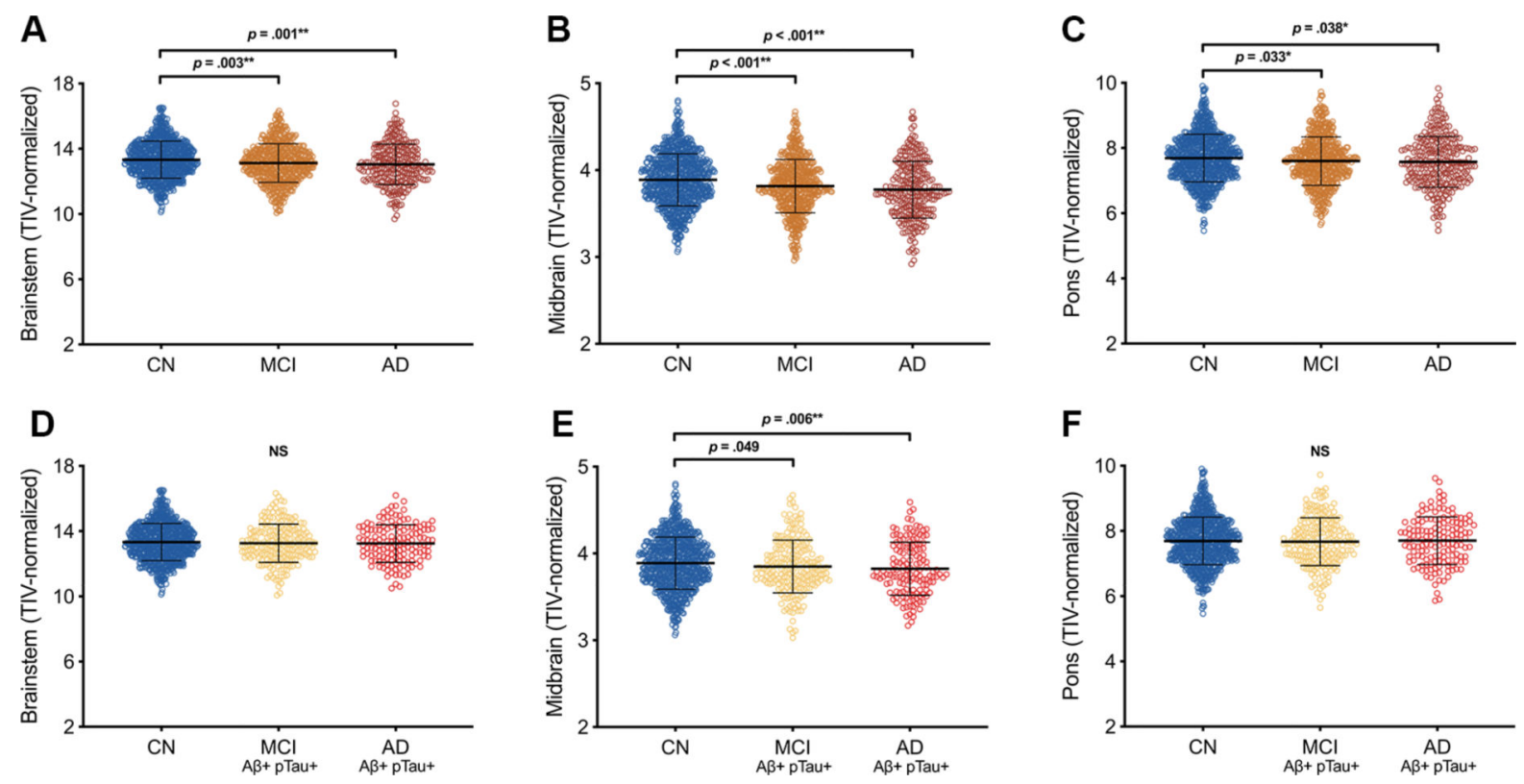

Figure 1. Brainstem gross regional volumes in cognitively normal, MCI, and Alzheimer's disease participants

Relative to older adults who are cognitively normal (CN; blue dots), those with mild cognitive impairment (MCI; orange dots) and clinically-diagnosed Alzheimer's disease dementia (AD; dark red dots) exhibit smaller volumes of overall brainstem (A), midbrain (B), and pons (C). In a subgroup with biomarker-confirmed AD pathology based on CSF $A \beta_{1-42}$ and pTau abnormalities, brainstem volume differences are specific to the midbrain in AD dementia (bright red dots) (D-F). All p-values reported are the results of post-hoc Fisher's LSD pairwise comparisons following one-way ANCOVA controlling for age, sex, education, and $A P O E \varepsilon 4$ status. *Remains significant with FDR limited to .10; **Remains significant with FDR limited to .05 or .10 . Error bars represent \pm 1 standard deviation. Abbreviations: $\mathrm{AD}=$ Alzheimer's disease $\mathrm{CN}=$ cognitively normal; $\mathrm{MCI}=$ mild cognitive impairment; NS = non-significant. 


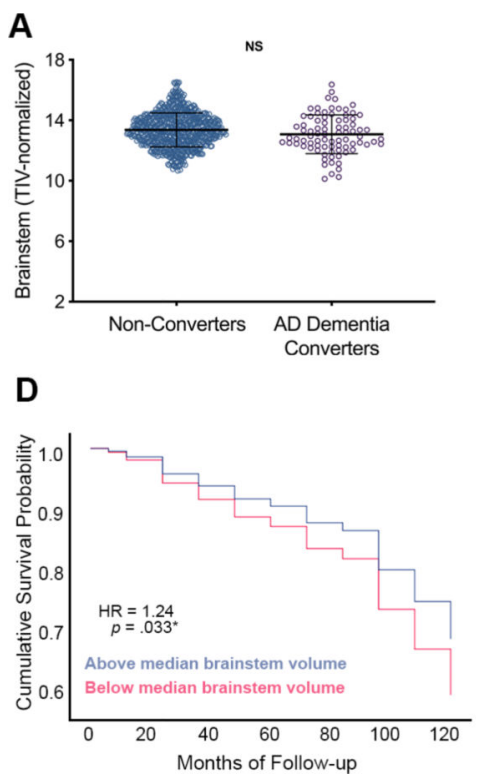

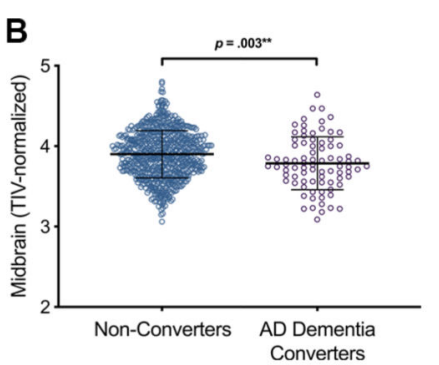

E

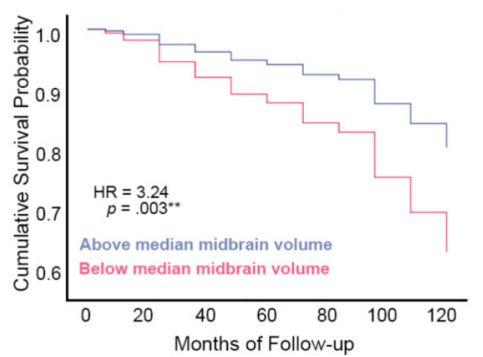

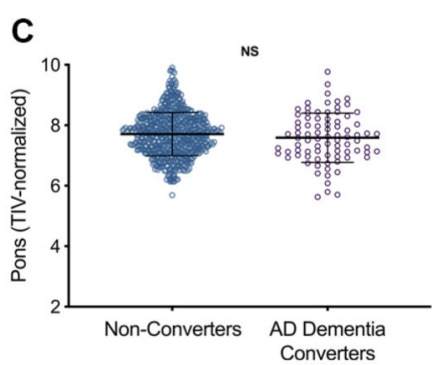

F

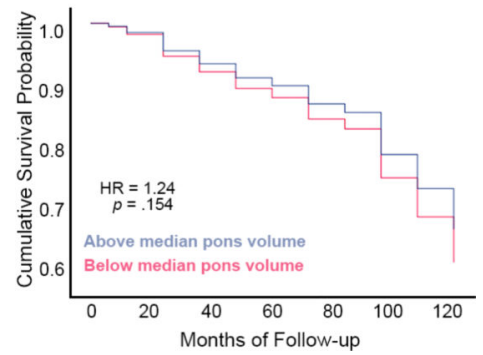

Figure 2. Brainstem gross regional volumes in progression to AD dementia $\mathrm{CN}$ older adults who prospectively progress to dementia (AD dementia converters; purple dots) demonstrated smaller volume of the midbrain (B) at baseline relative to those who did not progress to dementia (non-converters; blue dots), while no differences were observed in whole brainstem (A) and pons (C). $\mathrm{CN}$ older adults with smaller baseline midbrain volumes (pink lines) are more likely to later develop cognitive impairment and receive a clinical diagnosis of AD dementia over 6 to 120 months of follow-up than those with larger baseline midbrain volumes (blue lines) (D-F). Hazard ratios (HR) and $p$ values report results of proportional hazards Cox regressions with continuous brainstem ROI volume as predictor variable, $\mathrm{AD}$ dementia diagnosis as event of interest, and months to diagnosis as time variable (event cases $=83$; censored cases $=702$ ). ROI volume was first entered as a sole continuous predictor variable, and significant models added in covariates of age, sex, education, and $A P O E \varepsilon 4$ status. For display purposes, median splits were performed on brainstem ROI volumes to show risk at high and low volumes. *Remains significant with FDR limited to .10; **Remains significant with FDR limited to .05 or .10. Abbreviations: $\mathrm{AD}=$ Alzheimer's disease $; \mathrm{CN}=$ cognitively normal; $\mathrm{NS}=$ non-significant. 
A

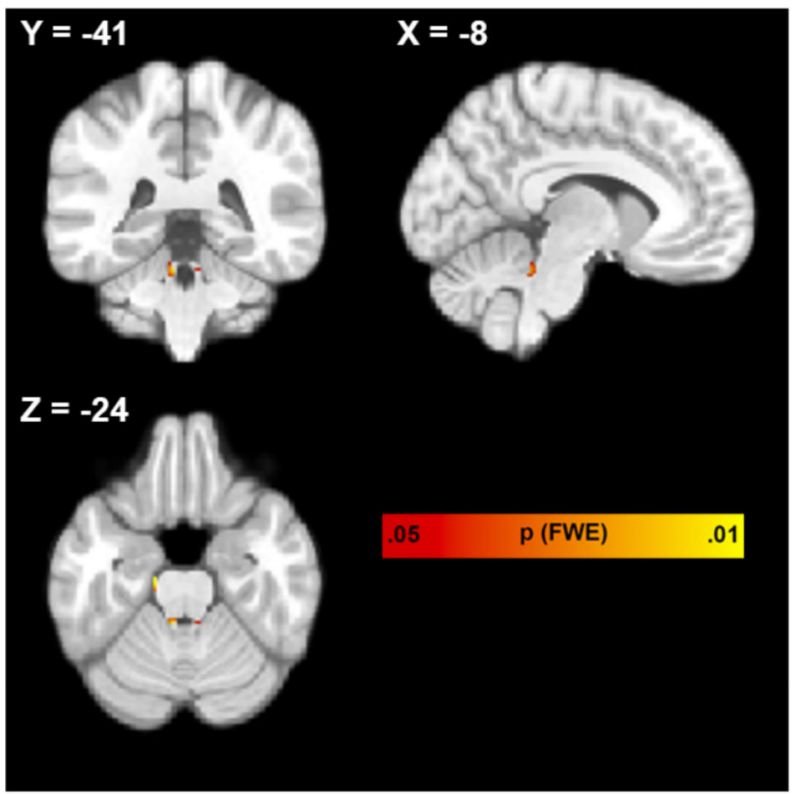

C

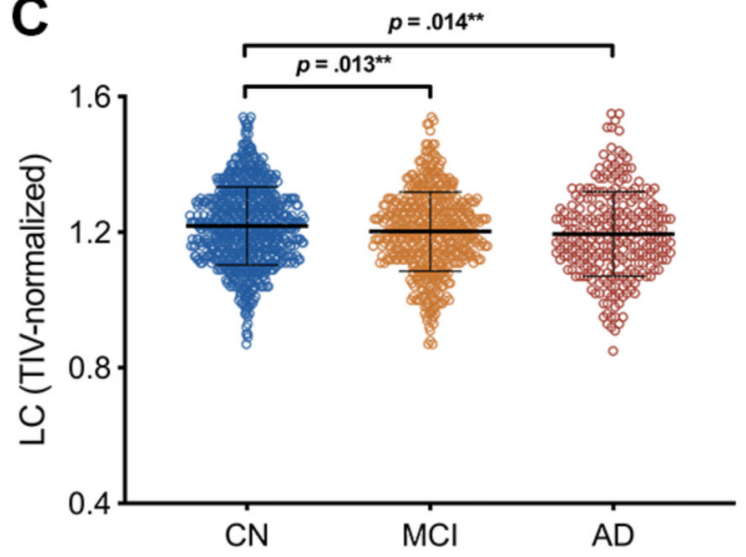

B
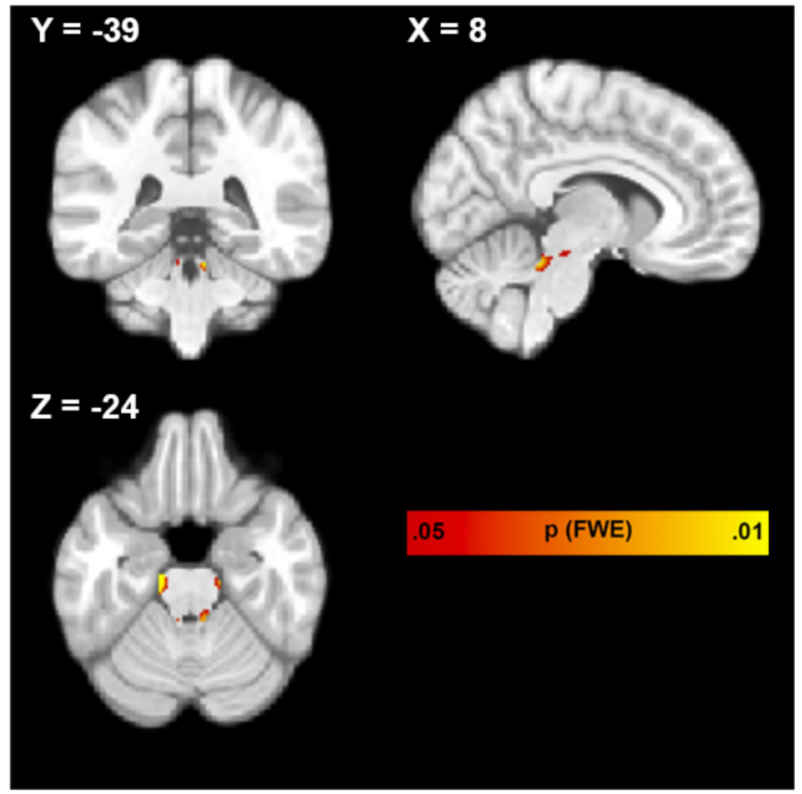

D

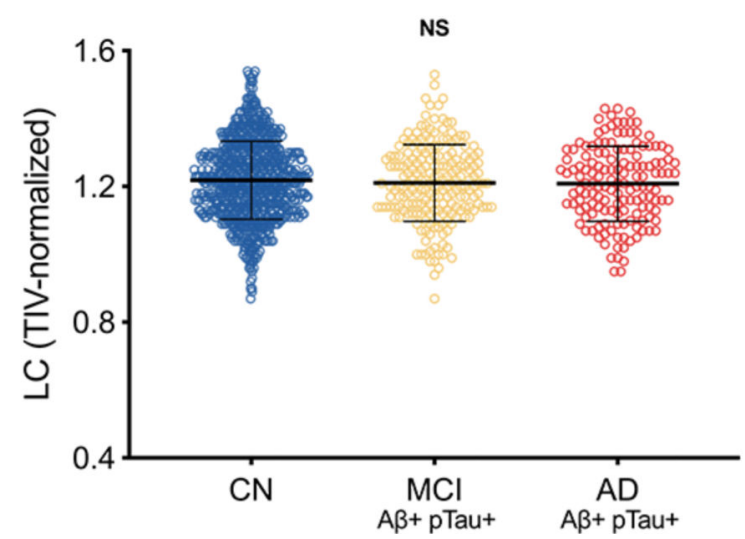

Figure 3. Brainstem-masked VBM comparisons and regional LC volumes

Brainstem-masked VBM analyses in the overall sample revealed a specific pattern of reduced regional dorsal rostral brainstem volume at baseline in MCI (A) and clinicallydiagnosed $\mathrm{AD}(\mathrm{B})$ relative to those who are $\mathrm{CN}$ that closely corresponds to the anatomical distribution of the LC. VBM analyses consisted of two-sample t-tests with age, sex, education, $A P O E \varepsilon 4$ status, and TIV as covariates. Results are displayed at FWE-corrected height threshold of $p<.05$, represented by color bar. Images are displayed in neurological orientation. LC ROI volumes, extracted using an LC atlas mask, (C) were smaller in MCI (orange dots) and AD (dark red dots) relative to cognitively normal older adults (blue dots). No significant differences were observed between biomarker-confirmed subgroups (D). $p$ values reported are the results of post-hoc Fisher's LSD pairwise comparisons following one-way ANCOVA controlling for age, sex, education, and APOE 44 status. *Remains significant with FDR limited to .10; **Remains significant with FDR limited to .05 or .10. 
Error bars represent \pm 1 standard deviation. Abbreviations: $\mathrm{AD}=$ Alzheimer's disease; $\mathrm{CN}=$ cognitively normal; $\mathrm{MCI}=$ mild cognitive impairment; $\mathrm{NS}$ = non-significant. 

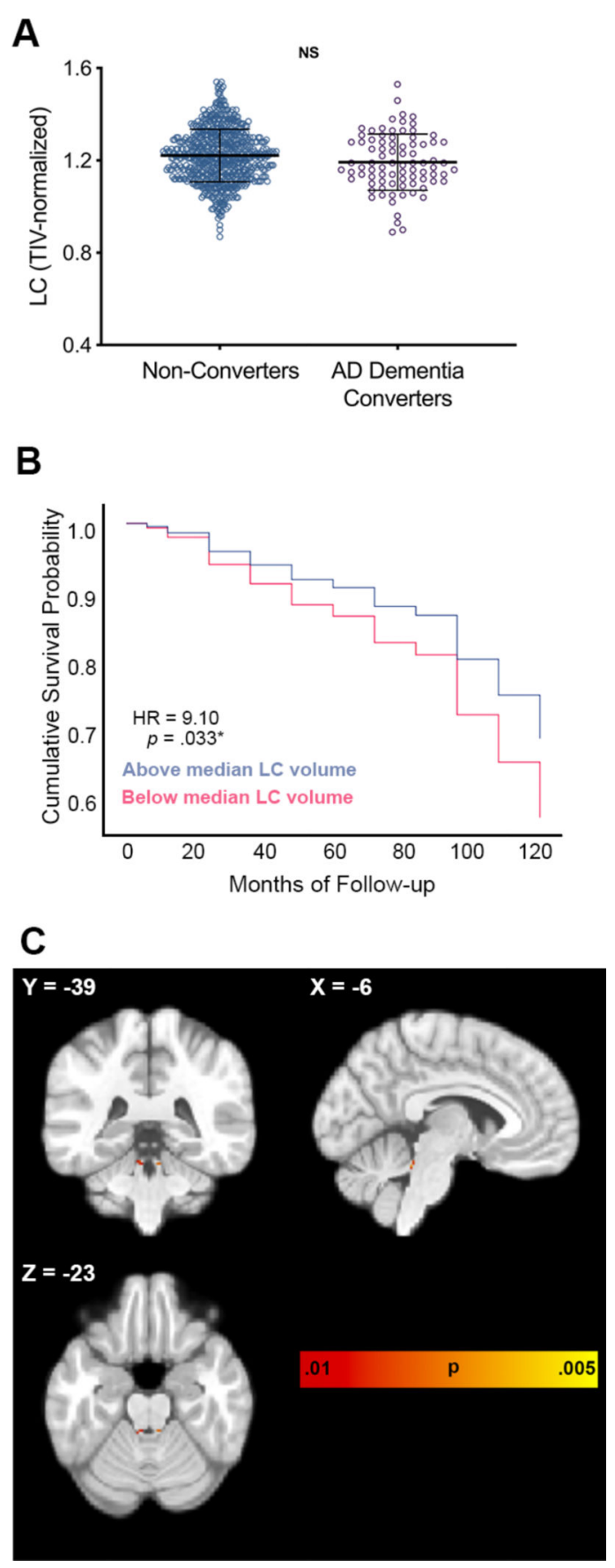

Figure 4. LC volume predicts progression to dementia in cognitively normal older adults AD dementia converters (purple dots) did not differ from non-converters (blue dots) in LC volume at baseline (A). CN older adults with smaller baseline LC volumes (pink lines) are more likely to later develop cognitive impairment and receive a clinical diagnosis of $\mathrm{AD}$ dementia over 6 to 120 months of follow-up than those with larger baseline midbrain volumes (blue lines) (B). Hazard ratios (HR) and p-values report results of proportional hazards Cox regressions with continuous brainstem ROI volume as predictor variable, AD dementia diagnosis as event of interest, and months to diagnosis as time variable (event 
cases $=83$; censored cases $=702$ ). ROI volume was first entered as a sole continuous predictor variable, and significant models added in covariates of age, sex, education, and $A P O E \varepsilon 4$ status. For display purposes, median split was performed on LC ROI volume to show risk at high and low volumes. Brainstem-masked VBM analyses revealed a specific pattern of reduced regional dorsal rostral brainstem volume at baseline in AD dementia converters compared to non-converters $(C)$ that closely corresponds to the anatomical distribution of the LC. VBM analyses consisted of two-sample t-tests with age, sex, education, $A P O E \varepsilon 4$ status, and TIV as covariates. VBM results are displayed at uncorrected height threshold of $p<.01$, represented by color bar. Images are displayed in neurological orientation. *Remains significant with FDR limited to .10. Error bars represent \pm 1 standard deviation. Abbreviations: $\mathrm{AD}=$ Alzheimer's disease; $\mathrm{CN}=$ cognitively normal; $\mathrm{L}=$ left; $\mathrm{NS}$ = non-significant; $\mathrm{R}=$ right. 
Table 1.

Baseline demographics and neuroimaging data for cognitively normal, MCI, and AD

\begin{tabular}{|c|c|c|c|c|c|c|}
\hline & $\mathrm{CN}$ & MCI & AD & $F$ or $\chi^{2}$ & $p$-value & $\eta_{p}^{2}$ or Cramer's V \\
\hline \multicolumn{7}{|l|}{ Demographics } \\
\hline$n$ & 814 & 542 & 273 & & & \\
\hline Age & 73.49 (6.76) & $73.54(7.35)$ & $75.12(7.74)$ & 5.78 & .003 & .007 \\
\hline $\operatorname{Sex}(M / F)$ & $417 / 397$ & $332 / 210$ & $153 / 120$ & 13.30 & .001 & .09 \\
\hline Education & $16.29(2.65)$ & $15.85(2.92)$ & $15.23(2.92)$ & 14.20 & $<.001$ & .017 \\
\hline$A P O E \varepsilon 4(0 / 1 / 2 \varepsilon 4)$ & $536 / 246 / 32$ & $249 / 221 / 72$ & $87 / 132 / 54$ & 138.36 & $<.001$ & .206 \\
\hline \multicolumn{7}{|l|}{ Neuroimaging $a, b$} \\
\hline TIV & $1499.35(146.12)$ & $1517.81(157.29)$ & 1504.57 (166.64) & 0.47 & .628 & .001 \\
\hline Brainstem & $13.33(1.14)$ & $13.13(1.18)$ & $13.05(1.24)$ & 7.13 & .001 & .009 \\
\hline Midbrain & $3.89(0.30)$ & $3.82(0.31)$ & $3.78(0.33)$ & 16.80 & $<.001$ & .02 \\
\hline Pons & $7.69(0.73)$ & $7.60(0.74)$ & $7.58(0.78)$ & 3.26 & .039 & .004 \\
\hline LC & $1.22(0.11)$ & $1.20(0.12)$ & $1.19(0.12)$ & 4.50 & .011 & .006 \\
\hline Demographics & $\mathrm{CN}$ & $\mathrm{MCI}[\mathrm{A} \beta+\mathrm{pTau}+]$ & $\mathrm{AD}[\mathrm{A} \beta+\mathrm{pTau}+]$ & & & \\
\hline$n$ & 814 & 202 & 145 & & & \\
\hline Age & $73.49(6.76)$ & $73.61(7.13)$ & $73.91(8.01)$ & 0.23 & .797 & $<.001$ \\
\hline $\operatorname{Sex}(M / F)$ & $417 / 397$ & $112 / 90$ & $78 / 67$ & 1.31 & .521 & .034 \\
\hline Education & $16.29(2.65)$ & $15.98(2.86)$ & $15.58(2.72)$ & 4.68 & .009 & .008 \\
\hline$A P O E \varepsilon 4(0 / 1 / 2 \varepsilon 4)$ & $536 / 246 / 32$ & $57 / 104 / 41$ & $31 / 77 / 37$ & 197.6 & $<.001$ & .292 \\
\hline \multicolumn{7}{|l|}{ Neuroimaging ${ }^{a, c}$} \\
\hline TIV & $1499.35(146.12)$ & $1505.68(166.34)$ & $1508.55(172.89)$ & 1.05 & .35 & .002 \\
\hline Brainstem & $13.33(1.14)$ & $13.26(1.17)$ & $13.24(1.15)$ & 0.98 & .376 & .002 \\
\hline Midbrain & $3.89(0.30)$ & $3.85(0.30)$ & $3.82(0.30)$ & 4.58 & .01 & .008 \\
\hline Pons & $7.69(0.73)$ & $7.67(0.73)$ & $7.70(0.73)$ & 0.15 & .859 & $<.001$ \\
\hline LC & $1.22(0.11)$ & $1.21(0.11)$ & $1.21(0.11)$ & 0.76 & .469 & .001 \\
\hline
\end{tabular}

Mean (standard deviation) are shown for all variables except for sex and $A P O E \varepsilon 4$. F or $\chi^{2}$ are result of one-way ANOVA (age, education), chisquare test of independence (sex, $A P O E \varepsilon 4$ ), or one-way ANCOVA (all other variables; covariates = age, sex, education, $A P O E \varepsilon 4)$. Effect sizes are displayed as $\eta_{p}{ }^{2}$ (age, education, neuroimaging variables) or Cramer's V (sex, APOE $\varepsilon 4$ ). Results from pairwise comparisons are displayed in Supplementary Table 1. Omnibus $p$-values for planned comparisons (neuroimaging variables) remained significant when FDR rate was limited to 0.05 (exception: pons $\mathrm{CN}$ vs. MCI vs. $\mathrm{AD}$ ) and 0.10 (all comparisons).

${ }^{a}$ TIV is presented in milliliters. ROI volumes were normalized using the following equation: (ROI volume / TIV) $\times 10^{3}$ for brainstem, midbrain, and pons or (ROI volume / TIV) x $10^{4}$ for LC

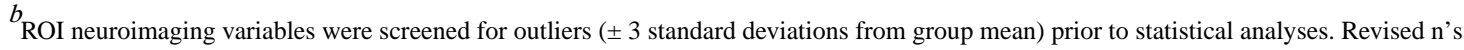
(CN/MCI/AD) by brainstem ROI are as follows: TIV(813/541/273); brainstem (809/541/272); midbrain (810/541/273); pons (810/541/272); LC $(808 / 542 / 273)$

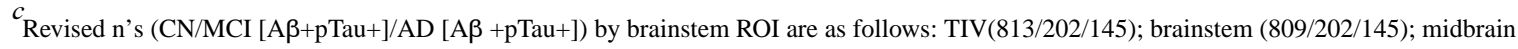
(810/202/145); pons (810/202/145); LC (808/202/145)

Abbreviations: $\mathrm{A} \beta$, amyloid $\beta$; $\mathrm{AD}$, Alzheimer's disease; $A P O E \varepsilon 4$, apolipoprotein $\varepsilon 4 ; \mathrm{CN}$, cognitively normal; $\mathrm{FDR}$, false discovery rate; $\mathrm{LC}$, locus coeruleus; MCI, mild cognitive impairment; pTau, phosphorylated tau; ROI, region-of-interest; TIV, total intracranial volume 
Table 2.

Baseline demographics and neuroimaging data for cognitively normal participants who progressed to dementia (converters) and did not progress to dementia (non-converters)

\begin{tabular}{|c|c|c|c|c|c|}
\hline & Non-converters & Converters & $F$ or $\chi^{2}$ & $p$-value & $\eta_{p}{ }^{2}$ or Cramer's V \\
\hline \multicolumn{6}{|l|}{ Demographics } \\
\hline$n$ & 702 & 83 & & & \\
\hline Age & $73.38(6.79)$ & 75.17 (6.29) & 5.18 & .023 & .007 \\
\hline $\operatorname{Sex}(M / F)$ & $350 / 352$ & $54 / 29$ & 6.87 & .009 & .094 \\
\hline Education & $16.36(2.68)$ & $15.71(2.40)$ & 4.50 & .034 & .006 \\
\hline$A P O E \varepsilon 4(0 / 1 / 2 \varepsilon 4)$ & $482 / 198 / 22$ & $35 / 38 / 10$ & 30.01 & $<.001$ & .196 \\
\hline \multicolumn{6}{|l|}{ Neuroimaging ${ }^{a} b$} \\
\hline TIV & $1498.76(147.91)$ & 1520.87 (132.93) & 0.16 & .688 & $<.001$ \\
\hline Brainstem & $13.36(1.12)$ & 13.07 (1.28) & 3.64 & .057 & .005 \\
\hline Midbrain & $3.90(0.29)$ & $3.79(0.33)$ & 8.68 & .003 & .011 \\
\hline Pons & $7.71(0.72)$ & $7.59(0.82)$ & 1.63 & .202 & .002 \\
\hline LC & $1.22(0.11)$ & $1.19(0.12)$ & 2.96 & .086 & .004 \\
\hline
\end{tabular}

Mean (standard deviation) are shown for all variables. $F$ or $\chi^{2}$ are result of one-way ANOVA (age, education), chi- square test of independence (sex, APOE ع4), or one-way ANCOVA (all other variables; covariates = age, sex, education, APOE ع4). Effect sizes are displayed as $\eta_{p}^{2}$ (age, education, neuroimaging variables) or Cramer's V (sex, APOE $\varepsilon 4$ ). All omnibus $p$-values for planned comparisons (neuroimaging variables) remained significant when FDR rate was limited to 0.05 and 0.10

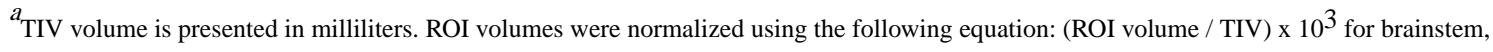
midbrain, and pons or (ROI volume / TIV) $\mathrm{x} 10^{4}$ for LC

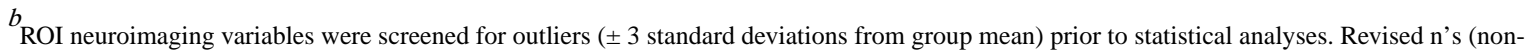
converters/converters) by brainstem ROI are as follows: TIV (701/83), brainstem (697/83), midbrain (698/83), pons (697/83), LC (696/83)

Abbreviations: $A P O E \varepsilon 4$, apolipoprotein $\varepsilon 4$; FDR, false discovery rate; LC, locus coeruleus; ROI, region-of-interest; TIV, total intracranial volume 
Table 3.

Cox regression models predicting dementia risk from baseline brainstem volumes

\begin{tabular}{|c|c|c|c|}
\hline & HR & $95 \% \mathrm{CI}$ & $p$-value \\
\hline \multicolumn{4}{|l|}{ Model 1a } \\
\hline Brainstem & 1.22 & {$[1.01,1.48]$} & .043 \\
\hline \multicolumn{4}{|l|}{ Model 1b } \\
\hline Brainstem & 1.24 & {$[1.02,1.52]$} & .033 \\
\hline Age & 1.03 & {$[1.00,1.07]$} & .085 \\
\hline Sex & 0.68 & {$[0.42,1.09]$} & .105 \\
\hline Education & 0.93 & {$[0.86,1.00]$} & .059 \\
\hline$A P O E \varepsilon 4$ & 2.76 & {$[1.98,3.84]$} & $<.001$ \\
\hline \multicolumn{4}{|l|}{ Model 2a } \\
\hline Midbrain & 3.05 & {$[1.47,6.32]$} & .003 \\
\hline \multicolumn{4}{|l|}{ Model 2b } \\
\hline Midbrain & 3.24 & {$[1.51,6.96]$} & .003 \\
\hline Age & 1.03 & {$[0.99,1.06]$} & .167 \\
\hline Sex & 0.70 & {$[0.44,1.12]$} & .139 \\
\hline Education & 0.92 & {$[0.85,1.00]$} & .047 \\
\hline$A P O E \varepsilon 4$ & 2.81 & {$[2.01,3.92]$} & $<.001$ \\
\hline \multicolumn{4}{|l|}{ Model 3} \\
\hline Pons & 1.24 & {$[0.92,1.68]$} & .154 \\
\hline \multicolumn{4}{|l|}{ Model 4a } \\
\hline LC & 9.16 & {$[1.32,63.55]$} & .025 \\
\hline \multicolumn{4}{|l|}{ Model 4b } \\
\hline LC & 9.10 & {$[1.20,69.22]$} & .033 \\
\hline Age & 1.03 & {$[0.99,1.07]$} & .107 \\
\hline Sex & 0.67 & {$[0.42,1.07]$} & .091 \\
\hline Education & 0.93 & {$[0.86,1.01]$} & .065 \\
\hline$A P O E \varepsilon 4$ & 2.74 & {$[1.97,3.82]$} & $<.001$ \\
\hline
\end{tabular}

Brainstem ROI volumes were reflected prior to Cox regression analyses to ensure consistent directionality, with smaller volume predicting greater risk. $p$-values for brainstem ROI variables were significant with FDR limited to 0.05 (midbrain) and 0.10 (brainstem, midbrain, LC). 